\title{
Muscle Alterations Are Associated With Minimal and Overt Hepatic Encephalopathy in Patients With Liver Cirrhosis
}

\author{
Silvia Nardelli iD , ${ }_{1}$ Barbara Lattanzi iD ${ }^{1}$ Manuela Merli, ${ }^{1}$ Alessio Farcomeni, ${ }^{2}$ Stefania Gioia (D),${ }^{1}$ Lorenzo Ridola, ${ }^{1}$ \\ and Oliviero Riggio ${ }^{1}$
}

\begin{abstract}
Muscle alterations (myosteatosis and sarcopenia) are frequent in cirrhosis and related to some complications including overt hepatic encephalopathy (HE). The aim of our study was to investigate the relationship between muscle alterations and minimal $\mathrm{HE}(\mathrm{MHE})$ and their role in the risk of overt HE. Sixty-four patients with cirrhosis were administered the Psychometric Hepatic Encephalopathy Score and animal naming test to detect MHE. Computed tomography was used to analyze the skeletal muscle index and attenuation. The incidence of the first episode of $\mathrm{HE}$, taking into account the competing risk nature of the data, was estimated. Myosteatosis was observed in 24 patients (37.5\%), sarcopenia in $37(58 \%)$, and $\mathrm{MHE}$ in 32 (50\%). Both myosteatosis $(62.5 \%$ versus $12.5 \%, P<0.001)$ and sarcopenia $(84 \%$ versus $31 \%, P<0.001)$ were more frequent in patients with MHE. The variables independently associated with the presence of MHE were sarcopenia, previous overt HE, and myosteatosis. Thirty-one (48\%) patients developed overt HE over $16.1 \pm 13$ months; myosteatosis was detected in $68 \%$ and sarcopenia in $84 \%$ of them. Sarcopenia and myosteatosis were also independently associated with the development of overt HE. Venous ammonia was significantly higher in patients with sarcopenia $(62.6 \pm 17.7$ versus $41.4 \pm 16.1 \mu \mathrm{g} / \mathrm{dL}, P<0.001)$ and in patients with myosteatosis $(65.2 \pm 19.2$ versus $46.7 \pm 17.1 \mu \mathrm{g} / \mathrm{dL}, P<0.001)$ and inversely correlated to both parameters. Survival was significantly lower in malnourished patients compared to patients without myosteatosis or sarcopenia $(P<0.001)$. Conclusion: Myosteatosis and sarcopenia, probably by reducing the handling of ammonia in the muscle, are independently associated with MHE and the risk of overt HE in patients with cirrhosis; in malnourished patients, the amelioration of nutritional status may be a goal to decrease both the prevalence of MHE and the incidence of overt HE. (Hepatology 2019;0:1-10).
\end{abstract}

$\mathrm{S}$ arcopenia is common in patients with cirrhosis, with a prevalence that has been reported to be as high as $65 \%-90 \% .{ }^{(1)}$ Multiple factors are involved in the genesis of sarcopenia: dietary intake is inadequate to meet energy expenditure, absorption is compromised, and substrate use is impaired due to liver disease. ${ }^{(2)}$ Muscular assessment with crosssectional imaging studies has become an attractive

index of nutritional status, ${ }^{(3,4)}$ and a recent European Consensus Statement has identified computed tomography (CT) as the gold standard for the detection of muscle wasting ${ }^{(5)}$ because it is not biased by the fluid overload frequently observed in decompensated cirrhosis. With this technique both quantitative and qualitative alterations in muscle tissue can be detected. Patients with cirrhosis may develop simultaneously

Abbreviations: ANT, animal naming test; BMI, body mass index; CT, computed tomography; HE, hepatic encephalopathy; HU, Hounsfield unit; MHE, minimal HE; PHES, Psychometric Hepatic Encephalopathy Score; SMI, skeletal muscle index; TIPS, transjugular intrahepatic portosystemic shunt.

Received January 16, 2019; accepted April 25, 2019.

Additional Supporting Information may be found at onlinelibrary.wiley.com/doi/10.1002/hep.30692/suppinfo.

(C) 2019 by the American Association for the Study of Liver Diseases.

View this article online at wileyonlinelibrary.com.

DOI 10.1002/hep.30692

Potential conflict of interest: Nothing to report. 
loss of skeletal muscle and gain of intermuscular and intramuscular fat, denominated "myosteatosis."(6) In populations without cirrhosis, myosteatosis increases with age and adiposity ${ }^{(7)}$ and is associated with metabolic abnormalities ${ }^{(8)}$ and decreased strength and mobility. ${ }^{(9)}$ In patients with liver cirrhosis, sarcopenia and myosteatosis may alter muscle strength and function and represent two independent prognostic factors for survival. ${ }^{(6,10)}$ Moreover, both conditions are associated with a higher prevalence of complications of liver cirrhosis ${ }^{(10)}$ including hepatic encephalopathy (HE). ${ }^{(11)}$ The rationale for a relationship between muscle depletion and $\mathrm{HE}$ derives from the possible involvement of muscle in ammonia metabolism and trafficking. In fact, as cirrhosis leads to a decline in the capacity of the liver to detoxify ammonia, skeletal muscle plays a compensatory role in ammonia metabolism and clearance, by the enzyme glutamine synthetase. ${ }^{(12)}$ Therefore, changes in muscle quantity and quality may lead to an increase in circulating ammonia levels and have relevant implications in favoring HE.

Minimal HE (MHE) is a subclinical cognitive impairment frequently observable in patients with cirrhosis detectable only by psychometry, ${ }^{(13,14)}$ which is strongly associated with the development of overt HE. ${ }^{(15,16)}$ Few studies investigated the relationship between sarcopenia and $\mathrm{MHE}^{(17,18)}$ in cirrhosis, and none of them used CT scanning to define muscle quantity and quality and MHE. More recently, a retrospective study showed a correlation between muscle alteration and history of overt HE. ${ }^{(11)}$ However, as MHE was not considered, the possibility that this relationship was actually due to a higher prevalence of MHE among the patients with muscle alterations cannot be ruled out.

Given this background, we hypothesized that the presence of sarcopenia and myosteatosis could be associated with the presence of $\mathrm{MHE}$ and, independently, with the risk of overt $\mathrm{HE}$ development during follow-up. A number of multivariate analyses were performed to establish the independent role of sarcopenia and myosteatosis in the occurrence of $\mathrm{MHE}$ as well as in the development of overt HE.

\section{Patients and Methods}

From September 2015 to June 2016, all patients with cirrhosis admitted into the Centre for the Study of Portal Hypertension in Rome with a CT scan performed as a standard of care during liver transplantation assessment or for the study of hepatic nodules were prospectively enrolled. The diagnosis of liver cirrhosis was based on clinical, biochemical, and radiological signs. At entry, overt $\mathrm{HE}$ was excluded by using a set of standardized closed questions based on the WestHaven criteria $^{(19)}$ (Supporting Information). Further exclusion criteria were alcohol/psychoactive drug intake (positive alcoholemia and/or benzodiazepine or opioid urine metabolites) at the moment of the psychometric evaluation, unrelated neurological disease, and lack of compliance with psychometric evaluation because of language barriers or reduced visual acuity. The presence of dementia was also excluded using a Mini-Mental State Examination score <26 despite its well-known limitation, ${ }^{(20)}$ as described. ${ }^{(21)}$ Patients with advanced hepatocellular carcinoma, outside the Milan criteria, were also excluded because in these patients the prognosis is strongly influenced by neoplastic disease. Patients with transjugular intrahepatic portosystemic shunt (TIPS) and/or large portosystemic shunts and patients with a history of persistent or recurrent $\mathrm{HE}$ defined by two or more than two episodes within the last 6 months, even if without overt $\mathrm{HE}$ on first observation, were also excluded because they are at higher risk of developing HE. A detailed clinical history was obtained in relation to previous

\section{ARTICLE INFORMATION:}

From the ${ }^{1}$ Department of Clinical Medicine, Centre for the Diagnosis and Treatment of Portal Hypertension; ${ }^{2}$ Department of Public Health and Infectious Diseases, "Sapienza" University of Rome, Rome, Italy.

\section{ADDRESS CORRESPONDENCE AND REPRINT REQUESTS TO:}

Silvia Nardelli, M.D., Ph.D.

Sapienza University of Rome, Gastroenterology
Viale dell'Università 37, 00161 Roma

E-mail: nardelli.silvia@gmail.com 
complications of liver cirrhosis, particularly previous episodes of overt HE. Patients were qualified as having a positive history if a previous episode of overt $\mathrm{HE}$ or grade 2 or higher $\mathrm{HE}$ (according to the West Haven criteria) was documented by a previous hospitalization. ${ }^{(22)}$ All the other parameters (Child-Pugh class and score, Model for End-Stage Liver Disease score, serum sodium and albumin levels) were collected during the enrollment hospitalization.

An informed, written consent for the collection and evaluation of demographic and clinical data was obtained. The "Sapienza" University of Rome Ethical Committee approved the study and allowed collection of the data (Rif.1720/01.10.09).

\section{BODY MASS INDEX CALCULATION AND CT SCAN EVALUATIONS}

Body mass index (BMI) was calculated to have a screening evaluation of the patient's nutritional status and used in the determination of the myosteatosis cutoff. ${ }^{(6)}$ In patients with ascites, the body weight for BMI calculation was detected in the absence of fluid retention (dry body weight) as recommended by the European Association for the Study of the Liver (EASL) guidelines on nutrition in chronic liver diseases. ${ }^{(23)}$ For the quantitative analysis of muscle mass, the transverse CT image at the L3 level was selected for each scan. All CT images were analyzed by two trained observers (B.L., S.N.), and images were analyzed with SliceOmatic V4.2 software (Tomovision, Montreal, Canada), which enables specific tissue demarcation using previously reported Hounsfield unit (HU) thresholds. ${ }^{(11)}$ Skeletal muscle is identified and quantified by $\mathrm{HU}$ thresholds of -29 to +150 , as described ${ }^{(24)}$; and with these specific HU thresholds, measurements of skeletal muscle index (SMI) are not influenced by the presence of ascites. Cross-sectional areas (square centimeters) were automatically computed by summing tissue pixels and multiplying by pixel surface area. Muscle cross-sectional area was normalized for stature (square centimeters per square meter) to obtain the L3 SMI. Sarcopenia was defined according to validated cutoff values in patients with cirrhosis: L3 SMI $<39 \mathrm{~cm}^{2} / \mathrm{m}^{2}$ for female patients and $<50 \mathrm{~cm}^{2} / \mathrm{m}^{2}$ for male patients. ${ }^{(25)}$ The following HU thresholds were used for adipose tissues: -190 to
-30 for subcutaneous and intermuscular adipose tissue $^{(26)}$ and -150 to -50 for visceral adipose tissue. ${ }^{(27)}$

Because muscle attenuation indirectly measures fat infiltration in muscles, mean muscle attenuation in HU was reported for the entire muscle area from the same image used to calculate L3 SMI. To define myosteatosis, we used cutoff values that have been associated with mortality: $<41 \mathrm{HU}$ in patients with a BMI up to 24.9 and $<33$ in those with a $\mathrm{BMI} \geq 2$. $^{(6,28)}$

\section{EVALUATION OF MHE}

The psychometric evaluation was performed in a quiet room, with no distracting noises. All patients underwent the Psychometric Hepatic Encephalopathy Score (PHES) battery, including the digit-symbol test, the trail-making tests $\mathrm{A}$ and $\mathrm{B}$, the serial-dotting test, and the line-tracing test. ${ }^{(29)}$ Each test was scored against age-adjusted and education-adjusted norms for the Italian population. The PHES is the sum of integer scores of each test computed from the adjusted $z$ values as follows: score -3 for $z \leq-3$, score -2 for $-3<z \leq-2$, score -1 for $-2<z \leq-1$, score 0 for $-1<z<1$, score 1 for $z \geq 1$. A PHES $\leq-4$ was considered abnormal. ${ }^{(30)}$ Moreover, to support the diagnosis of $\mathrm{MHE}$, all patients underwent the animal naming test (ANT) that consists of the maximum number of animals listed in 1 minute, as recently described. ${ }^{(31)}$ Patients were considered to be affected by MHE when two tests (PHES + ANT) were abnormal, according to EASL/American Association for the Study of Liver Diseases guidelines. ${ }^{(32)}$ The assessors of MHE were blinded to the results of the nutritional assessment.

\section{FOLLOW-UP}

All patients were offered follow-up in the outpatient department with repeated ultrasound and laboratory investigations every 6 months and endoscopic evaluation every 1 or 2 years. Patients and their families were instructed on the importance of adhering to the scheduled visits and to contact the medical staff immediately should any alteration in the mental status occur between scheduled reviews. In particular, the family was instructed to report the occurrence of lethargy, apathy, obvious personality change, inappropriate behavior, or disorientation in 
time and place, which correspond to the occurrence of a grade 2 alteration in the patient's mental status. In this case, $\mathrm{HE}$ evaluation including the psychometric performance was repeated by medical staff to confirm and stage the degree of HE. Patients with an overt episode of HE reached the main endpoint of the study. Patients were contacted by phone every 3 months to check on their adherence to the scheduled follow-up. Patients were followed up until death, liver transplantation, or the last available outpatient review.

\section{STATISTICAL ANALYSIS}

The data are reported as mean $\pm \mathrm{SD}$. Comparisons between groups were performed by unpaired Student $t$ or chi-squared test. Logistic regression analysis was used to identify clinical and biochemical variables independently and significantly associated to $\mathrm{MHE}$, a variable that is not time-dependent. We estimated the cumulative incidence of the first episode of $\mathrm{HE}$ (time-dependent variable) during the follow-up, taking into account the nature of the competing risks in the data (HE before liver transplantation, death, and liver transplantation are competing events). Additionally, the conditional subdistribution hazard at multivariate analysis was evaluated using the model of Fine and Gray. ${ }^{(33)}$ The factors associated with the development of $\mathrm{HE}$ were initially evaluated by univariate models (using univariate Fine and Gray models) and then included in multivariate analyses (according to multivariate Fine and Gray models). In our final multivariate models we included one covariate per 10 events for $\mathrm{MHE}$ and one per 15 events for the incidence of overt HE. Final models were chosen using forward stepwise selection by minimizing the Akaike information criterion. The final multivariate logistic regression model was also compared with Firth bias-reduced penalized-likelihood logistic regression, where we found similar results. We report the results of Firth bias-reduced regression. Finally, in order to deal with possible confounding, we also estimated overparameterized multivariate models in which we included all measured possible confounders. Because the number of covariates included in that model was large, we bias-corrected the estimates using a nonparametric bootstrap procedure based on 1,000 replicates. Software R, version 3.4.2, was used for all computations.

\section{Results}

Of the 105 patients with cirrhosis admitted to our department from September 2015 to June 2016, some were excluded: 15 for the presence of overt $\mathrm{HE}$ (grade 2 or more), 5 for positive alcoholemia, 6 for psychoactive drug intake, 10 for altered Mini-Mental State Examination $(<26)$ or neurological diseases, and 5 for lack of compliance with psychometric tests. The demographic, clinical, and biochemical characteristics of all patients enrolled in the study are reported in Table 1.

Sarcopenia according to SMI cutoff was present in 37 patients (58\%); compared to the patients without sarcopenia, patients with muscle depletion had more often a previous history of $\mathrm{HE}$ (43\% versus $15 \%$, $P=0.02)$ and were more often classified as affected by $\mathrm{MHE}$ ( $73 \%$ versus $18.5 \%, P<0.001$ ), according to the PHES. Venous ammonia was higher in patients with sarcopenia than in patients without sarcopenia $(62.6 \pm 17.7$ versus $41.4 \pm 16.1 \mu \mathrm{g} / \mathrm{dL}, P<0.001)$. Moreover, muscle attenuation was significantly lower in patients with sarcopenia than in patients without sarcopenia $(29.9 \pm 8.1$ versus $37.5 \pm 7.7 \mathrm{HU}, P<0.001)$.

According to the cutoff based on muscle attenuation and BMI, myosteatosis was present in 24 patients (37.5\%). Compared to the patients without myosteatosis, patients with fatty infiltration of the muscle

TABLE 1. Demographic and Clinical Characteristics of the Patients Enrolled in the Study

\begin{tabular}{lc} 
& Patients $(\mathrm{n}=64)$ \\
\hline Sex (M/F) & $48 / 16$ \\
Age (years) & $56.7 \pm 10.1$ \\
Etiology (virus/alcohol/NASH/other) & $45 / 13 / 4 / 2$ \\
MELD & $13.9 \pm 5.6$ \\
Child-Pugh class (A/B/C) & $20 / 33 / 11$ \\
Child-Pugh score & $7.7 \pm 1.8$ \\
Previous HE (no/yes) & $44 / 20$ \\
Gastrointestinal bleeding (no/yes) & $50 / 14$ \\
Ascites (no/yes) & $28 / 36$ \\
Hemoglobin (g/dL) & $11.6 \pm 2.2$ \\
Bilirubin (mg/dL) & $3.3 \pm 6.1$ \\
Albumin (g/dL) & $3.3 \pm 0.6$ \\
INR & $1.5 \pm 0.3$ \\
Sodium (mEg/L) & $135.7 \pm 4.2$ \\
BMl (kg/m²) & $22.8 \pm 3.8$
\end{tabular}

Abbreviations: INR, international normalized ratio; MELD, Model for End-Stage Liver Disease; NASH, nonalcoholic steatohepatitis. 

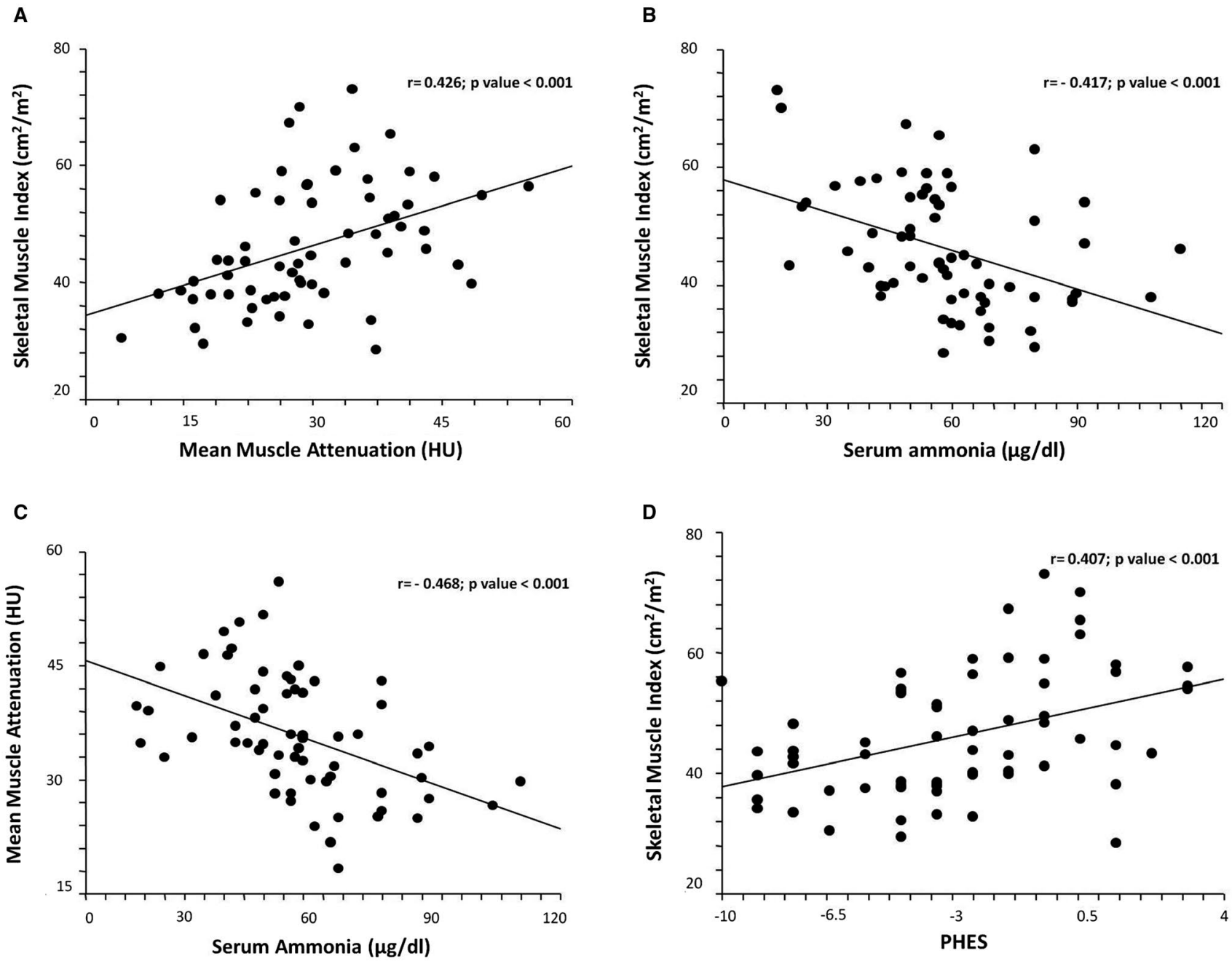

FIG. 1. Pearson's correlations between SMI and muscle attenuation (A), SMI and ammonia (B), muscle attenuation and ammonia (C), and SMI and PHES (D).

had lower albumin $(2.9 \pm 0.6$ versus $3.4 \pm 0.6 \mathrm{~g} / \mathrm{dL}$, $P=0.006)$ and sodium $(134.2 \pm 4.9$ versus $136.6 \pm 3.4$ $\mathrm{mEq} / \mathrm{L}, P=0.03)$ levels and were more often classified as affected by MHE ( $83 \%$ versus 50\%, $P<0.001$ ), according to the PHES. Venous blood ammonia was higher in patients with myosteatosis $(65.2 \pm 19.2$ versus $46.7 \pm 17.1 \mu \mathrm{g} / \mathrm{dL}, P<0.001)$.

Statistically significant correlations were observed between SMI and muscle attenuation (direct, Fig. 1A), SMI and ammonia (inverse, Fig. 1B), and ammonia and muscle attenuation (inverse, Fig. 1C), indicating that low muscle mass and high fat infiltration correspond to high ammonia levels.

MHE was diagnosed at entry and observed in half of the patients. The comparison between patients with and without MHE is reported in Table 2. Patients with MHE, compared to those without, had a higher prevalence of ascites and overt $\mathrm{HE}$ in the past. Notably, venous blood ammonia was significantly higher as was the presence of sarcopenia or myosteatosis in patients with MHE than in those without. Finally, a significant correlation was found between SMI and PHES (Fig. 1D).

In order to establish the relative role of the variables significantly associated with $\mathrm{MHE}$ at the univariate analysis and taking into consideration the prevalence of MHE in our sample (for details, see Statistical Analysis above), we performed two multivariate analyses, both reported in Table 3 . In the first model the variables independently associated with 
TABLE 2. Comparison of Demographic and Clinical Characteristics Between Patients With and Without MHE

\begin{tabular}{lccc} 
& $\mathrm{MHE}^{-}(\mathrm{n}=32)$ & $\mathrm{MHE}^{+}(\mathrm{n}=32)$ & $P$ \\
\hline Sex (M/F) & $24 / 8$ & $24 / 8$ & 1 \\
Age (years) & $56.2 \pm 9.7$ & $57.2 \pm 10.5$ & 0.69 \\
Etiology (virus/alcohol/other) & $21 / 6 / 5$ & $24 / 7 / 1$ & 0.22 \\
MELD & $13.3 \pm 5.2$ & $14.2 \pm 5.7$ & 0.48 \\
Child-Pugh class (A/B/C) & $15 / 12 / 5$ & $6 / 21 / 5$ & 0.06 \\
Child-Pugh score & $7.4 \pm 1.9$ & $8 \pm 1.7$ & 0.14 \\
Previous HE (no/yes) & $28 / 4$ & $16 / 16$ & 0.001 \\
Ascites (no/yes) & $18 / 14$ & $9 / 23$ & 0.02 \\
Gastrointestinal bleeding & $24 / 7$ & $25 / 6$ & 0.75 \\
$\quad$ (no/yes) & & & \\
Bilirubin (mg/dL) & $2.5 \pm 2.6$ & $4.1 \pm 8.2$ & 0.30 \\
Albumin (g/dL) & $3.5 \pm 0.5$ & $3 \pm 0.6$ & 0.004 \\
INR & $1.5 \pm 0.4$ & $1.5 \pm 0.4$ & 0.83 \\
Sodium (mEq/L) & $136.6 \pm 3.9$ & $134.8 \pm 4.3$ & 0.09 \\
NH3 ( $\mu$ g/dL) & $43.5 \pm 16.3$ & $63.8 \pm 18.1$ & $<0.001$ \\
ANT (no. animals) & $15.3 \pm 5.2$ & $11.4 \pm 2.5$ & $<0.001$ \\
SMl (cm $/ \mathrm{m}^{2}$ ) & $50.7 \pm 10.9$ & $41.8 \pm 7.7$ & $<0.001$ \\
Sarcopenia (no/yes) (\%) & $22 / 10(31)$ & $5 / 27(84)$ & $<0.001$ \\
Muscle attenuation (HU) & $37.2 \pm 8.1$ & $29.1 \pm 7.4$ & $<0.001$ \\
Myosteatosis (no/yes) (\%) & $28 / 4(12.5)$ & $12 / 20(62.5)$ & $<0.001$
\end{tabular}

Mean \pm SD.

Abbreviations: INR, international normalized ratio; MELD, Model for End-Stage Liver Disease; NASH, nonalcoholic steatohepatitis; $\mathrm{NH} 3$, serum ammonia.

the presence of MHE were sarcopenia, myosteatosis, and history of previous overt HE (Table 3). In the second model we included all known and measured possible confounders and bias-corrected the estimates using a nonparametric bootstrap procedure based on 1,000 replicates. In the second model sarcopenia and previous overt $\mathrm{HE}$ were significantly related to $\mathrm{MHE}$, while the relations between myosteatosis and $\mathrm{MHE}(P=0.053)$ and low albu$\min (P=0.052)$ were at the limit of significance (Table 3).

During a mean follow-up of $14.9 \pm 12.5$ months, 31 patients (48\%) experienced grade 2 or higher HE. A precipitating factor was identified in 25 patients (80\%): 12 episodes (48\%) were caused by infections, $6(24 \%)$ by diuretic overdose and electrolyte disorders, 4 (16\%) by gastrointestinal bleeding, and $3(12 \%)$ by constipation. On multivariate analysis, sarcopenia and myosteatosis were independently associated with the development of overt $\mathrm{HE}$ according to the first model (Table 4). In the second model, we included all known and measured possible confounders and bias-corrected
TABLE 3. Results of Multivariate Analyses Predicting MHE

\begin{tabular}{lcccc} 
& OR & Cl Lower & Cl Upper & $P$ \\
\hline Firth bias-reduced logistic regression analysis & & \\
Sarcopenia & 5.33 & 1.53 & 20.43 & 0.008 \\
Previous overt HE & 5.48 & 1.33 & 26.97 & 0.018 \\
Myosteatosis & 7.54 & 1.97 & 34.32 & 0.002 \\
Overparameterized logistic regression analysis after bias reduction using \\
bootstrap \\
MELD score & 0.95 & 0.81 & 1.06 & 0.314 \\
Sarcopenia & 2.87 & 1.02 & 22.23 & 0.046 \\
Previous overt HE & 2.69 & 1.16 & 25.03 & 0.030 \\
Myosteatosis & 2.51 & 0.97 & 22.23 & 0.053 \\
NH3 & 1.01 & 0.98 & 1.07 & 0.266 \\
Albumin & 0.39 & 0.05 & 1.01 & 0.052
\end{tabular}

Abbreviations: CI, confidence interval; MELD, Model for EndStage Liver Disease; NH3, serum ammonia; OR, odds ratio.

TABLE 4. Results of Multivariate Analyses Predicting Overt HE

\begin{tabular}{lcccc} 
& SHR & Cl lower & Cl upper & $P$ \\
\hline Firth bias-reduced multivariate analysis & & & \\
Sarcopenia & 21.55 & 2.55 & 181.93 & 0.005 \\
Myosteatosis & 5.47 & 1.88 & 15.95 & 0.002
\end{tabular}

Overparameterized multivariate analysis after bias reduction using bootstrap

$\begin{array}{lcccc}\text { MELD score } & 0.95 & 0.87 & 1.05 & 0.38 \\ \text { Sarcopenia } & 18.3 & 2.82 & 29.3 & 0.005 \\ \text { Previous overt HE } & 2.15 & 1.03 & 5.26 & 0.04 \\ \text { Myosteatosis } & 1.29 & 0.45 & 3.81 & 0.60 \\ \text { NH3 } & 0.99 & 0.96 & 1.01 & 0.60 \\ \text { MHE } & 2.60 & 0.75 & 13.0 & 0.11 \\ \text { Albumin } & 0.35 & 0.13 & 0.94 & 0.03\end{array}$

Mean \pm SD .

Abbreviations: CI, confidence interval; MELD, Model for EndStage Liver Disease; NH3, serum ammonia; OR, odds ratio; sHR, subdistribution hazard ratio.

the estimates using a nonparametric bootstrap procedure based on 1,000 replicates. In the second model sarcopenia, previous overt $\mathrm{HE}$, and low albumin were significantly related to overt HE (Table 4).

During the episode, patients with grade 2 or higher HE were often unable to complete the PHES, and they were graded according to the standardized closed questions (21 patients experienced grade 2, 8 patients grade 3 , and 2 patients grade 4 ). In 25 of 31 patients who experienced overt HE, PHES was repeated after the resolution of overt $\mathrm{HE}$ and $\mathrm{MHE}$ was observed in 12 patients (48\%). 
The incidence of $\mathrm{HE}$, taking into account as a competitive risk death or liver transplantation, was significantly higher in patients with sarcopenia or myosteatosis than in those without (Figs. 2 and 3).

During the same follow-up, 25 patients died (39\%). The main causes of death were infections and sepsis

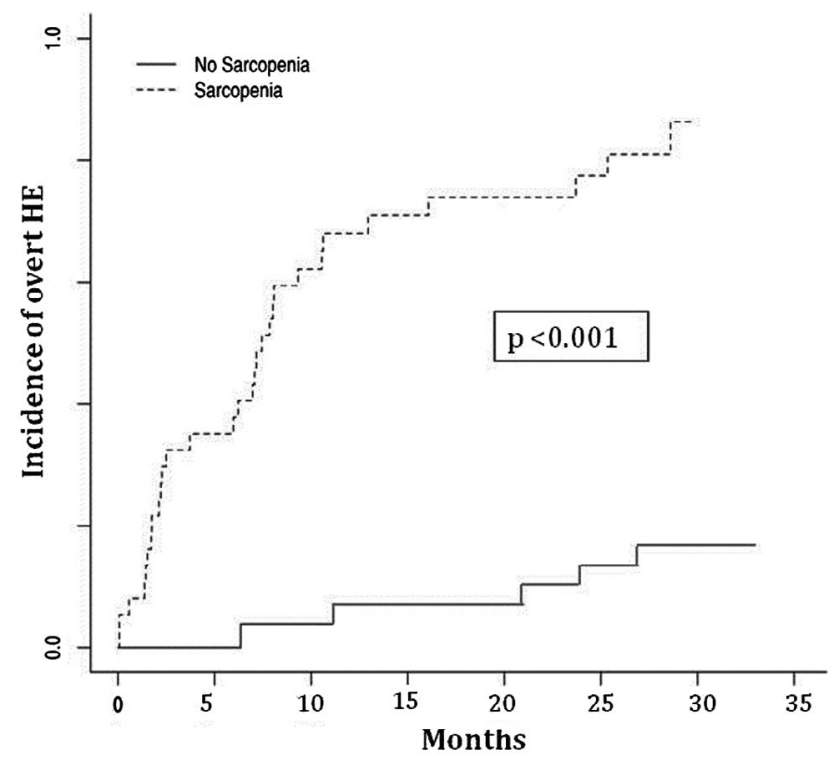

FIG. 2. Cumulative incidence of overt $\mathrm{HE}$ among patients with and without sarcopenia.

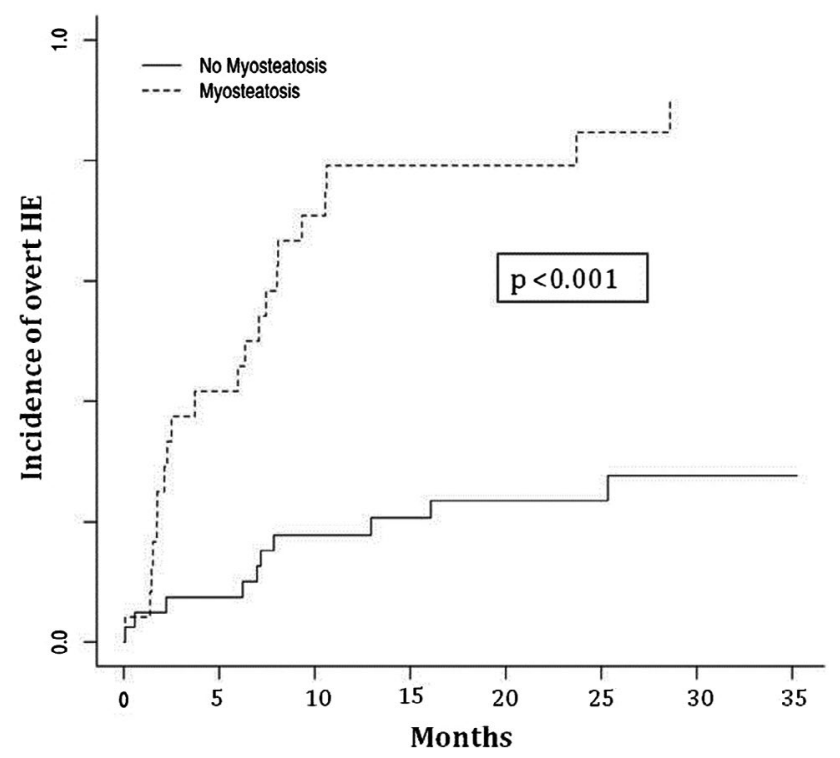

FIG. 3. Cumulative incidence of overt $\mathrm{HE}$ among patients with and without myosteatosis.
(33\%), liver failure (36\%), variceal bleeding (12\%), and other causes not related to liver disease (19\%). The overall survival was significantly lower in patients with sarcopenia than in those without $(36 \%$ versus $64 \%, P<0.001$ ), as well as in patients with myosteatosis compared to those without (10\% versus $90 \%$, $P<0.001)$.

\section{Discussion}

In this study we investigated the association between myosteatosis and MHE, and we confirm a strong correlation between muscle alterations and cognitive impairment in patients with cirrhosis. Skeletal muscle abnormalities are frequently observed in patients with cirrhosis ${ }^{(1)}$ and are associated with more clinical complications ${ }^{(10,11)}$ and worse survival $^{(6)}$ compared to patients with cirrhosis who do not have muscle alterations.

In patients with cirrhosis, the relationship between malnutrition, survival, and occurrence of complications has been known for a long time. ${ }^{(34)}$ Especially protein malnutrition is important in this relationship. However, the study of protein malnutrition in patients with cirrhosis is difficult, especially in the advanced stage of the disease. Methods based on the estimation of muscle wasting, such as anthropometry, have been shown to be more accurate. ${ }^{(35)}$ In our study, sarcopenia was diagnosed by CT scan, which is now considered one of the tools to optimally quantify the skeletal muscle mass and for the identification of patients with sarcopenia and cirrhosis. ${ }^{(36-38)}$ Although related to other diagnostic tools such as dual-energy X-ray absorptiometry and mid-arm muscle circumference (MAMC) determined anthropometrically, CT scanning is able to identify a larger number of patients with sarcopenia and cirrhosis compared to other methods. ${ }^{(39)}$ Moreover, CT scanning, by using muscle attenuation, is indirectly able to measure muscle fat infiltration and to add information not only on quantity but also on the quality of muscle tissue. Similar results can be obtained by evaluating the fat-free muscle mass using magnetic resonance, as recently reported in patients with cirrhosis subjected to TIPS. ${ }^{(40)}$ When estimation of muscle function is required, assessment of muscle quality cannot be eluded, especially because patients with sarcopenic obesity have been described. The use of BMI, although limited in patients with cirrhosis 
by the possibility of fluid retention, is considered a reliable tool to identify obese patients and to correct the data derived by the CT examination in order to obtain cutoff values for myosteatosis. In our samples none of the patients was classified as obese according to the BMI values.

The association between sarcopenia and $\mathrm{HE}$ seems to be more complex than previously thought. The more straightforward interpretation is that muscle is an alternative site for ammonia detoxification, when the liver is impaired by cirrhosis. Thus, it follows that the presence of sarcopenia leads to a higher risk of $\mathrm{HE} .^{(17,41)}$ However, more recently, it has been suggested that ammonia may itself contribute to sarcopenia in patients with cirrhosis by interfering with protein remodeling, generating a vicious cycle where sarcopenia promotes $\mathrm{HE}$ and hyperammonemia and hyperammonemia further worsens sarcopenia. ${ }^{(42)}$ In order to confirm this hypothesis, a recent animal model showed that treatment of hyperammonemia improved skeletal muscle strength and mass. ${ }^{(43)}$ Also, in our study, higher levels of ammonia were found in patients with MHE and in malnourished patients.

The relationship between myosteatosis and $\mathrm{HE}$ is even less clear than that between sarcopenia and HE. One hypothesis is that fat infiltration, by reducing the fat-free mass, may contribute to the reduction of ammonia removal by glutamine synthetase, which is supposed to be a function of muscle cells. The significant correlation reported in Fig. 3 between ammonia, SMI, and muscle attenuation seems to support this hypothesis. Another possibility is that intermuscular and intramuscular adipose tissue may contribute to increasing the inflammatory cytokines, which has been described in patients with cirrhosis and is supposed to increase the ammonia toxicity. ${ }^{(44)}$

Previous studies have examined the potential role of muscle wasting and function in the neurocognitive alterations in patients with cirrhosis. Handgrip strength, used to assess muscle function in 84 patients with cirrhosis, was found to be an independent predictor of complications, including $\mathrm{HE}^{(16)}$ In a prospective study of 300 patients with cirrhosis, we found that muscle depletion, detected by MAMC, and muscle function, detected by handgrip strength, were related to clinically overt $\mathrm{HE}$ at admission and in the last 12 months as well as to the presence of covert $\mathrm{HE}$ detected by the PHES. ${ }^{(17)}$ Both these observations are confirmed in the present study, in which muscle wasting was detected by CT scan, which is considered the gold standard.

Although few studies have investigated the relationship between sarcopenia and minimal and overt $\mathrm{HE}^{(15-18)}$ in cirrhosis, only one retrospective study analyzed the correlation between myosteatosis and a history of $\mathrm{HE}^{(11)}$ and no study has evaluated the link between myosteatosis and MHE. In this study we found that myosteatosis is an independent risk factor for the presence of MHE and for the development of overt HE. The main limitations of our study are the small sample size and the selection bias of including only patients with cirrhosis with a CT scan. Moreover, the relationship between muscle wasting and $\mathrm{HE}$ seen in the present study is based on statistical correlations and would be better supported by the demonstration that improvement of sarcopenia and myosteatosis may lead to improvement of a patient's cognitive function. Another limitation of the study is that we did not have a second CT scan at follow-up and a consequent psychometric evaluation, and thus, we are not able to relate the modification of muscle parameters that eventually occurred in patients with cirrhosis with modification of the cognitive impairment. The relationship between the evolution of muscle wasting and the patients' cognitive impairment would be very interesting and could better support the association between malnutrition and $\mathrm{HE}$. We have recently published a paper on the modification of muscle mass and the evolution of HE after a TIPS, a procedure able to spontaneously ameliorate the muscle mass, at least in some patients. In this setting we described that the patients with a muscle wasting amelioration after TIPS placement obtained an improvement of the cognitive impairment and a lower number of episodes of overt $\mathrm{HE}$ in the follow-up, ${ }^{(45)}$ supporting a causal relationship between muscle alterations and HE. Unfortunately, at the moment, the amelioration of muscle wasting independent of that occurring in some patients after a TIPS for still unknown reasons is hard to obtain in patients with cirrhosis.

In conclusion, our study indicates that muscle wasting and muscle quality are strongly associated with the presence of MHE and favors the development of $\mathrm{HE}$ in patients with cirrhosis. The mechanisms linking muscle alteration to $\mathrm{HE}$ are probably related to the failure in ammonia handling by the wasting muscles, which probably reduces their capacity to increase 
glutamine synthesis from ammonia. A consequence of these observations is that measures to avoid malnutrition should be considered to prevent this complication. The possibility of reducing the burden of $\mathrm{HE}$ as well as other complications of liver cirrhosis by improving a patient's nutritional status needs further studies.

\section{REFERENCES}

1) Merli M, Giusto M, Gentili F, Novelli G, Ferretti G, Riggio $O$, et al. Nutritional status: its influence on the outcome of patients undergoing liver transplantation. Liver Int 2010;30: 208-214.

2) Plauth M, Merli M, Kondrup J, Weimann A, Ferenci P, Müller MJ; ESPEN Consensus Group. ESPEN guidelines for nutrition in liver disease and transplantation. Clin Nutr 1997;16:43-55.

3) Montano-Loza AJ. New concepts in liver cirrhosis: clinical significance of sarcopenia in cirrhotic patients. Minerva Gastroenterol Dietol 2013;59:173-186.

4) Montano-Loza AJ. Muscle wasting: a nutritional criterion to prioritize patients for liver transplantation. Curr Opin Clin Nutr Metab Care 2014;17:219-225.

5) Cruz-Jentoft AJ, Baeyens JP, Bauer JM, Boirie Y, Cederholm T, Landi F, et al. Sarcopenia: European consensus on definition and diagnosis: report of the European Working Group on Sarcopenia in Older People. Age Ageing 2010;39:412-423.

6) Montano-Loza AJ, Angulo P, Meza-Junco J, Prado CM, Sawyer $\mathrm{MB}$, Beaumont C, et al. Sarcopenic obesity and myosteatosis are associated with higher mortality in patients with cirrhosis. J Cachexia Sarcopenia Muscle 2016;7:126-135.

7) Gallagher D, Kuznia P, Heshka S, Albu J, Heymsfield SB, Goodpaster B, et al. Adipose tissue in muscle: a novel depot similar in size to visceral adipose tissue. Am J Clin Nutr 2005;81: 903-910.

8) Petersen KF, Befroy D, Dufour S, Dziura J, Ariyan C, Rothman $\mathrm{DL}$, et al. Mitochondrial dysfunction in the elderly: possible role in insulin resistance. Science 2003;300:1140-1142.

9) Visser M, Goodpaster BH, Kritcheversusky SB, Newman AB, Nevitt M, Rubin SM, et al. Muscle mass, muscle strength, and muscle fat infiltration as predictors of incident mobility limitations in well-functioning older persons. J Gerontol A Biol Sci Med Sci 2005;60:324-333.

10) Eslamparast T, Montano-Loza AJ, Raman M, Tandon $P$. Sarcopenic obesity in cirrhosis- the confluence of 2 prognostic titans. Liver Int 2018;38:1706-1717.

11) Bhanji RA, Moctezuma-Velazquez C, Duarte-Rojo A, Ebadi M, Ghosh S, Rose C, et al. Myosteatosis and sarcopenia are associated with hepatic encephalopathy in patients with cirrhosis. Hepatol Int 2018;12:377-386.

12) Olde Damink SWM, Jalan R, Redhead DN, Hayes PC, Deutz NE, Soeters PB. Inter organ ammonia and amino acid metabolism in metabolically stable patients with cirrhosis and a TIPSS. Hepatology 2002;37:1163-1170.

13) Weissenborn $K$, Giewekemeyer $K$, Heidenreich $S$, Bokemeyer M, Berding G, Ahl B. Attention, memory, and cognitive function in hepatic encephalopathy. Metab Brain Dis 2005;20:359-367.

14) Amodio P, Del Piccolo F, Marchetti P, Angeli P, Iemmolo R, Caregaro L, et al. Clinical features and survival of cirrhotic patients with subclinical cognitive alterations detected by the number connection test and computerized psychometric tests. Hepatology 1999;29:1662-1667.

15) Riggio O, Amodio P, Farcomeni A, Merli M, Nardelli S, Pasquale C, et al. A model for predicting development of overt hepatic encephalopathy in patients with cirrhosis. Clin Gastroenterol Hepatol 2015;13:1346-1352.

16) Nardelli S, Gioia S, Pasquale C, Pentassuglio I, Farcomeni A, Merli M, et al. Cognitive impairment predicts the occurrence of hepatic encephalopathy after transjugular intrahepatic portosystemic shunt. Am J Gastroenterol 2016;111:523-528.

17) Kalaitzakis E, Olsson R, Henfridsson P, Hugosson I, Bengtsson $\mathrm{M}$, Jalan R, et al. Malnutrition and diabetes mellitus are related to hepatic encephalopathy in patients with liver cirrhosis. Liver Int 2007;27:1194-1201.

18) Merli M, Giusto M, Lucidi C, Giannelli V, Pentassuglio I, Di Gregorio V, et al. Muscle depletion increases the risk of overt and minimal hepatic encephalopathy: results of a prospective study. Metab Brain Dis 2013;28:281-284.

19) Montagnese S, Russo FP, Amodio P, Burra P, Gasbarrini A, Loguercio C, et al. Hepatic encephalopathy 2018: a clinical practice guideline by the Italian Association for the Study of the Liver (AISF). Dig Liver Dis 2019;51:190-205.

20) Corrias M, Turco M, Rui MD, Gatta A, Angeli P, Merkel C, et al. Covert hepatic encephalopathy: does the Mini-Mental State Examination help? J Clin Exp Hepatol 2014;4:89-93.

21) Nardelli S, Gioia S, Pasquale C, Pentassuglio I, Farcomeni A, Merli M, et al. Cognitive impairment predicts the occurrence of hepatic encephalopathy after transjugular intrahepatic portosystemic shunt. Am J Gastroenterol 2016;111:523-528.

22) Lucidi C, Ginanni Corradini S, Abraldes JG, et al. Hepatic encephalopathy expands the predictivity of Model for End-Stage Liver Disease in liver transplant setting: evidence by means of 2 independent cohorts. Liver Transpl 2016;22:1333-1342.

23) European Association for the Study of the Liver. EASL Clinical Practice Guidelines on nutrition in chronic liver disease. J Hepatol 2019;70:172-193.

24) Mitsiopoulos N, Baumgartner RN, Heymsfield SB, Lyons W, Gallagher D, Ross R. Cadaver validation of skeletal muscle measurement by magnetic resonance imaging and computerized tomography. J Appl Physiol 1998;85:115-122.

25) Carey EJ, Lai JC, Wang CW, Dasarathy S, Lobach I, MontanoLoza AJ, et al. A multicenter study to define sarcopenia in patients with end-stage liver disease. Liver Transpl 2017;23:625-633.

26) Kvist H, Sjostrom L, Tylen U. Adipose tissue volume determinations in women by computed tomography: technical considerations. Int J Obes 1986;10:53-67.

27) Vehmas T, Kairemo KJ, Taavitsainen MJ. Measuring visceral adipose tissue content from contrast enhanced computed tomography. Int J Obes Relat Metab Disord 1996;20:570-573.

28) Martin L, Birdsell L, Macdonald N, Reiman T, Clandinin MT, $\mathrm{McC}$ argar LJ, et al. Cancer cachexia in the age of obesity: skeletal muscle depletion is a powerful prognostic factor, independent of body mass index. J Clin Oncol 2013;31:1539-1547.

29) Weissenborn K, Ennen JC, Schomerus H, Ruckert N, Hecker H. Neuropsychological characterization of hepatic encephalopathy. J Hepatol 2001;34:768-773.

30) Amodio P, Campagna F, Olianas S, Iannizzi P, Mapelli D, Penzo M, et al. Detection of minimal hepatic encephalopathy: normalization and optimization of the psychometric hepatic encephalopathy score. A neuropsychological and quantified EEG study. J Hepatol 2008;49:346-353.

31) Campagna F, Montagnese S, Ridola L, Senzolo M, Schiff S, De Rui M, et al. The animal naming test: An easy tool for the assessment of hepatic encephalopathy. Hepatology 2017;66:198-208.

32) Vilstrup H, Amodio P, Bajaj J, Cordoba J, Ferenci P, Mullen KD, et al. Hepatic encephalopathy in chronic liver disease: 2014 Practice Guideline by the American Association for the Study of Liver Diseases and the European Association for the Study of the Liver. Hepatology 2014;60:715-735. 
33) Fine JP, Gray RJ. A proportional hazards model for the subdistribution of a competing risk. J Am Stat Assoc 1999;94:496-509.

34) Child CG III, Turcotte JG. Surgery and portal hypertension. In: Child CG, ed. The Liver and Portal Hypertension. Philadelphia, PA: W.B. Saunders Co. 1964:50-64.

35) Merli M, Romiti A, Riggio O, Capocaccia L. Optimal nutritional indexes in chronic liver disease. JPEN J Parenter Enteral Nutr 1987;11(5 Suppl.):130S-134S.

36) Montano-Loza AJ, Meza-Junco J, Prado CM, Lieffers JR, Baracos VE, Bain VG, et al. Muscle wasting is associated with mortality in patients with cirrhosis. Clin Gastroenterol Hepatol 2012;10:166-173.

37) Tandon P, Ney M, Irwin I, Ma MM, Gramlich L, Bain VG, et al. Severe muscle depletion in patients on the liver transplant wait list: its prevalence and independent prognostic value. Liver Transpl 2012;18:1209-1216.

38) DiMartini A, Cruz RJ Jr., Dew MA, Myaskoversusky L, Goodpaster B, Fox K, et al. Muscle mass predicts outcomes following liver transplantation. Liver Transpl 2013;19:1172-1180.

39) Giusto M, Lattanzi B, Albanese C, Galtieri A, Farcomeni A, Giannelli V, et al. Sarcopenia in liver cirrhosis: the role of computed tomography scan for the assessment of muscle mass compared with dual-energy X-ray absorptiometry and anthropometry. Eur J Gastroenterol Hepatol 2015;27:328-334.

40) Praktiknjo M, Book M, Luetkens J, Pohlmann A, Meyer C, Thomas D, et al. Fat-free muscle mass in magnetic resonance imaging predicts acute-on-chronic liver failure and survival in decompensated cirrhosis. Hepatology 2018;67:1014-1026.

41) Nardelli S, Lattanzi B, Torrisi S, Greco F, Farcomeni A, Gioia $\mathrm{S}$, et al. Sarcopenia is risk factor for development of hepatic encephalopathy after transjugular intrahepatic portosystemic shunt placement. Clin Gastroenterol Hepatol 2017;15:934-936.

42) Dasarathy S, Merli M. Sarcopenia from mechanism to diagnosis and treatment in liver disease. J Hepatol 2016;65:1232-1244.

43) Kumar A, Davuluri G, Silva RNE, Engelen MPKJ, Ten Have GAM, Prayson R, et al. Ammonia lowering reverses sarcopenia of cirrhosis by restoring skeletal muscle proteostasis. Hepatology 2017;65:2045-2058.

44) Aldridge DR, Tranah EJ, Shawcross DL. Pathogenesis of hepatic encephalopathy: role of ammonia and systemic inflammation. J Clin Exp Hepatol 2015;5(Suppl. 1):S7-S20.

45) Gioia S, Merli M, Nardelli S, Lattanzi B, Pitocchi F, Ridola $\mathrm{L}$, et al. The modification of quantity and quality of muscle mass improves the cognitive impairment after TIPS. Liver Int 2019;39:871-877.

\section{Supporting Information}

Additional Supporting Information may be found at onlinelibrary.wiley.com/doi/10.1002/hep.30692/suppinfo. 